\title{
Ueber die Vertheilung der Knoten der Planeten- und Cometèn-Bahnen.
}

Wenn auf der Peripherie eines in $m$ gleiche $\mathbf{A b}$. schnitte getheilten Kreises $n$ Punkte zufällig. d. h. so vertheilt sind, dass für sie jede Lage gleich wahrscheinlich ist, so ist die Wahrscheinlichkeit, dass sich solche Abschnitte vorfinden, welche $i$ Punkte enthalten, gleich

$$
\frac{n !}{i !(n-i) !}\left(\frac{1}{m}\right)^{i}\left(1-\frac{1}{m}\right)^{n-i}
$$

und die wahrscheinliche Zahl solcher Abschnitte ist

$$
m \frac{n !}{i !(n-i) !}\left(\frac{1}{m}\right)^{i}\left(\mathrm{I}-\frac{1}{m}\right)^{n-i}
$$

Von dieser Formel ausgehend, kann man die Frage beantworten, ob die Knoten der Asteroiden. oder CometenBahnen auf der Ekliptik zufallig vertheilt sind, oder ob ihre Vertheilung irgend einem Gesetze - etwa einer Condensation in gewissen bevorzugten Gegenden - unterworfen ist, denn jede Abweichung von einer zufäligen Vertheilung wird sich gleich aus ${ }^{\circ}$ dem Vergleiche zwischen den theoretischen nach obiger Formel berechneten Zahlen der Knotenpunkte in verschiedenen Theilen der Ekliptik und den thatsächlichen ergeben. So würde z. B. eine Con. densation um ein oder mehrere Centren die Zahl der leeren und der dichter besetzten Theilungsstücke auf Kosten derjenigen vermehren, welche nur einen oder wenige Punkte enthalten. Da nun eine positive oder negative Antwort auf die Frage über die Zufalligkeit der Vertheilung der Knoten als Beweismittel für oder gegen einige Hypothesen über die Entstehung des Asteroiden-Ringes dienen kann, so habe ich es unternommen, ihre Vertheilung in dieser Hinsicht zu untersuchen.

Die Bahnelemente von 250 Asteroiden, welche in dem Verzeichnisse des Annuaire du bureau des longitudes pour l'an 1886 angegeben sind, wurden auf einzelne Kärtchen geschrieben und dann nach den Knotenlängen geordnet. Die Ekliptik theilte ich in 180 Sectoren $z \mathrm{zu}$ je 2 Grad und zählte die in jedem Sector enthaltenen Planetenbahnen. Ich erhielt auf diese Weise folgende Zahlenreihe: $\left.{ }^{*}\right)$

$\begin{array}{lllllllll}14524 & 20101 & 03221 & 31112 & 23212 & 10110 & 03231 & 11150 & 31321 \\ 02000 & 12020 & 10100 & 02303 & 10231 & 12621 & 22024 & 31211 & 32122 \\ 20332 & 01110 & 44243 & 13201 & 11001 & 00101 & 10120 & 11002 & 10201 \\ 01010 & 10100 & 12010 & 10113 & 22211 & 11212 & 21320 & 04113 & 13312\end{array}$

Nun hat man aber nach der oben mitgetheilten Formel bei einer zufälligen Vertheilung von $25^{\circ}$ Punkten in 180 Intervallen eine Anzahl

$$
m_{0}=180\left(\frac{179}{180}\right)^{250}
$$

solcher Intervalle zu erwarten, welche keinen Punkt enthalten. Ebenso ist die wahrscheinliche Zahl derjenigen Abschnitte, in welchen $1,2 \ldots i$ Punkte enthalten sind, gleich

$$
\begin{aligned}
& m_{1}=180.250\left(\frac{179}{180}\right)^{249} \cdot \frac{1}{180}=\frac{250}{179} \cdot m_{0} \\
& m_{2}=\frac{249}{179} \cdot \frac{1}{2} m_{1} \\
& \ldots \ldots \ldots \ldots \ldots \\
& m_{i}=\frac{251-i}{179} \cdot \frac{1}{i} m_{i-1}
\end{aligned}
$$

Aus diesen Formeln kann man also die wahrscheinliche Zahl der 0 , der 1 , etc. in der oben geschriebenen Zahlenreihe ermitteln. Vergleicht man die so erhaltenen theoretischen Werthe mit den Resultaten einer directen Zählung, so erhält man folgende Zusammenstellung:

*) Da die Knoten-Längen in Zehntel Grad aufgeschrieben wurden, so bedeutet $z$. B. die erste Zahl, dass sich ein Knoten in dem Abschnitte 0.0 bis inclusive 1.9 , also zwischen den Längen 359.95-1.95, befindet. Ebenso sind 4 Knoten in dem Abschnitte 2.0-3.9, d. h. zwischen den Lungen 1.95-3.95, enthalten u. s. w.

\begin{tabular}{l|l|l|l|l}
\hline Punkte & Zählg. & Rechn. & Diff. & W. Grenzen \\
\hline
\end{tabular}

I. Zahl der Abschnitte $\left(m_{i}\right)$ :

\begin{tabular}{rr|r|r|r|r} 
Zahl der 0 & 46 & 44.7 & +1.3 & \pm 4.4 \\
1 & 63 & 62.4 & +0.6 & \pm 5.3 \\
2 & 40 & 43.4 & -3.4 & \pm 4.4 \\
3 & 21 & 20.1 & +0.9 & \pm 3.0 \\
4 & 7 & 6.9 & +0.1 & \pm 1.7 \\
5 & 2 & 1.9 & +0.1 & \pm 0.9 \\
6 und mehr & 1 & 0.6 & +0.4 & \pm 0.5
\end{tabular}

2. Zahl der Bahnen $\left(i m_{i}\right)$ :

\begin{tabular}{rr|r|r|r|r} 
je I & 63 & 62.4 & +0.6 & \pm 5.3 \\
2 & 80 & 86.8 & -6.8 & \pm 8.8 \\
3 & 63 & 60.3 & +2.7 & \pm 9.0 \\
4 & 28 & 27.6 & +0.4 & \pm 6.8 \\
5 & 10 & 9.5 & +0.5 & \pm 4.5 \\
6 und mehr & 6 & 3.4 & +2.6 & \pm 3.0
\end{tabular}

Unter der Ueberschrift $* W$. Grenzene sind in der letzten Vertikalreihe die Grössen

$$
8 / 3 \sqrt{m\left(\frac{m_{0}}{m}\right)\left(1-\frac{m_{0}}{m}\right)} \quad \text { resp. } 2 / 3 i \sqrt{m\left(\frac{m_{0}}{m}\right)\left(1-\frac{m_{0}}{m}\right)}
$$

angegeben, welche den wahrscheinlichen Fehler der $m_{i}$ bez. $i m_{i}$ vorstellen. Man sieht, dass die Uebereinstimmung 
zwischen Theorie und Erfahrung eine vollständige ist. Keine einzige Grösse uberschreitet ihre wahrscheinlichen Grenzen; wir können also auf Grund dieses Vergleiches eine

vollständige Zufalligkeit der Vertheilung behaupten.

der Knoten der Asteroiden-Bahnen

Fast ebenso verhält es sich mit den Cometen. Ich habe das Verzeichniss der Cometen-Bahnen dem Lexicon der Astronomie von Gretschel entnommen und es nach derselben Methode bearbeitet. Dabei wurden 273 seit dem Anfange der christlichen Zeitrechnung beobachtete nicht periodische Cometen in Rechnung gezogen. Die Vertheilung ihrer Knoten auf der Ekliptik wird durch folgende Zahlenreihe dargestellt.

$\begin{array}{llll}11201 & 04211 & 11411 & 44110 \\ 24231 & 01222 & 10113 & 22311 \\ 10323 & 21110 & 12160 & 21310 \\ 03103 & 11001 & 01112 & 01101\end{array}$

Zählt man nun in dieser Reihe die Nullen, die Einheiten etc., und vergleicht ihre Zahl mit einer auf obiger Formel basirten theoretischen Berechnung, so erhält man folgende Uebersicht.

\begin{tabular}{l|l|l|l|l}
\hline Punkte & Zählg. & Rechn. & Diff. & W. Grenzen \\
\hline
\end{tabular}

1. Zahl der Abschnitte :

\begin{tabular}{r|r|r|r|r} 
Zahl der 0 & 35 & 39.3 & -4.3 & \pm 4.2 \\
I & 73 & 60.0 & +13.0 & \pm 5.2 \\
2 & 37 & 45.6 & -8.6 & \pm 4.5 \\
3 & 21 & 23.0 & -2.0 & \pm 3.2 \\
4 & 10 & 8.7 & +1.3 & \pm 2.0 \\
5 & $I$ & 2.7 & -1.7 & \pm 1.1 \\
6 und mehr & 3 & 0.7 & +2.3 & \pm 0.6
\end{tabular}

2. Zahl der Bahnen:

\begin{tabular}{r|r|r|r|r} 
je I & 73 & 60.0 & +13.0 & \pm 5.2 \\
2 & 74 & 91.2 & -17.2 & \pm 9.0 \\
3 & 63 & 69.0 & -6.0 & \pm 9.6 \\
4 & 40 & 34.8 & +5.2 & \pm 8.0 \\
5 & 5 & 13.5 & -8.5 & \pm 5.5 \\
6 und mehr & 18 & 4.2 & +13.8 & \pm 3.0
\end{tabular}

Obgleich hier die Differenzen grösser ausfallen, so ist doch die Uebereinstimmung der Theorie mit der Beobachtung eine genügende, so dass man auch die Vertheilung der Knoten der Cometen-Bahnen als zufällig anzusehen hat.

$\mathrm{Um}$ ein Beispiel einer $\mathrm{nicht}$ zufäligen Vertheilung zu geben, welches im Stande wäre, die ausserordentliche Uebereinstimmung der aus der Wahrscheinlichkeitsrechnung gefundenen mit den thatsächlichen Grössen im Falle der Planeten und Cometen besser hervorzuheben, will ich noch hier die Vertheilung der Knoten der Meteorbahnen in Bezug auf ihre Zufälligkeit untersuchen.

Die Knoten der I 89 Meteorbahnen, welche Schiaparelli in seiner »Astronomischen Theorie der Sternschnuppen * aus den Beobachtungen von Zezioli hergeleitet hat, ergaben folgende Zahlenreihe (wegen der kleineren Zahl der Bahnen wurde die Ekliptik in 120 statt 180 Intervalle getheilt):

$\begin{array}{llllll}12111 & 21311 & \text { III42 } & 22222 & & \\ 50121 & 00022 & 11301 & 13330 & & \\ 31231 & 00102 & 63020 & 40122 & & \\ 13421 & 11031 & 10041 & 21230 & & \\ & & & & & \\ 10114 & 02260 & 04021 & 00011 & 26001 & 01101 \\ 00215 & 32279 & 45934 & 26000 & 01002 & 11011 \\ 11001 & 02004 & 21000 & 15211 & 11200 & 50100 \\ 40200 & 21306 & 31243 & 24054 & 10000 & 00001\end{array}$

und daraus erhalten wir:

\begin{tabular}{|c|c|c|c|c|}
\hline Punkte & Zählg. & Rechn. & Diff. & W. Gren \\
\hline & \multicolumn{4}{|c|}{ 1. Zahl der Abschnitte: } \\
\hline \multirow[t]{5}{*}{ Zahl der } & 47 & 24.7 & $+19 \cdot 3$ & $\pm 3 \cdot 3$ \\
\hline & 30 & 39.2 & -9.2 & \pm 4.2 \\
\hline & 17 & 31.0 & -14.0 & \pm 3.7 \\
\hline & 5 & 16.2 & -11.2 & \pm 2.7 \\
\hline & 9 & $6: 3$ & +2.7 & \pm 1.7 \\
\hline \multirow{3}{*}{6 und meh } & 5 & 2.0 & +3.0 & \pm 0.9 \\
\hline & 7 & 0.6 & +6.4 & \pm 0.5 \\
\hline & 120 & I 20.0 & & \\
\hline
\end{tabular}

2. Zahl der Bahnen:

\begin{tabular}{r|r|r|r|r} 
je 1 & 30 & 39.2 & -9.2 & \pm 4.2 \\
2 & 34 & 62.0 & -28.0 & \pm 7.4 \\
3 & 15 & 48.6 & -33.6 & \pm 8.1 \\
4 & 36 & 25.2 & +10.8 & \pm 6.8 \\
5 & 25 & 10.0 & +15.0 & \pm 4.5 \\
6 und mehr & 49 & 4.0 & +45.0 & \pm 3.5
\end{tabular}

Die theoretischen, nach dem Gesetze des Zufalles berechneten Werthe stimmen hier gar nicht mit den thatsächlichen überein. Keine einzige Grösse bleibt in ihren wahrscheinlichen Grenzen. Die Zahl der leeren Intervalle, sowie diejenige der dicht besetzten ist verhältnissmässig zu gross, woraús man auf die Existenz einer CondensationsUrsache schliessen darf. Dieses ist auch der Fall. Denn wir wissen, dass die Bewegung der Erde eine jährliche Periodicität der Menge der sichtbaren Sternschnuppenbahnen, und infolge dessen auch eine Verdichtung der Knoten in gewissen Gegenden der Ekliptik hervorbringt.

Dass eine ähnliche Verdichtung bei den Planetenbahnen gar nicht vorhanden ist, kann man auch dadurch beweisen, dass man die Entfernungen der Knoten der Planetenbahnen von einander berechnet und die Vertheilung dieser Entfernungen mit derjenigen vergleicht, welche bei 
einer zufälligen Vertheilung der Knotenpunkte stattfinden würde. Wäre nämlich irgendwo in der Ekliptik eine Verdichtung vorhanden, so würde dementsprechend die Zahl der kleinen Entfernungen merklich zunehmen, und ebenso die Zahl der sehr grossen, während die mittleren Entfernungen weniger häufig vorkommen würden. Dieses ist aber keineswegs der Fall, wie wir es gleich sehen werden.

Wir wollen erst die theoretische Zahl der Entfernungen von verschiedener Grösse bestimmen.

Befindet sich irgend ein Punkt in $M$, so ist die Wahrscheinlichkeit, dass in einem Bogenelemente $\mathrm{d} s$, welches in der Entfernung $s$ von $M$ liegt, ein beliebiger anderer von den übrigen $n-1$ Punkten enthalten ist, und dass dabei die Strecke $s$ von dem ersten Punkte in $M$ bis zum zweiten Punkte leer ist, gleich

$$
(n-\mathrm{I}) \frac{\mathrm{d} s}{2 \pi}\left(\mathrm{I}-\frac{s}{2 \pi}\right)^{n-2}
$$

Folglich ist die Wahrscheinlichkeit, dass sich leere Intervalle finden, deren Länge kleiner ist als $l$ gleich

$$
\frac{n-1}{2 \pi} \int_{0}^{l}\left(\mathrm{I}-\frac{s}{2 \pi}\right)^{n-2} \mathrm{~d} s=\mathrm{I}-\left(\mathrm{I}-\frac{l}{2 \pi}\right)^{n-1}
$$

und die wahrscheinliche Zahl solcher Entfernungen ist

$$
n\left[\mathrm{I}-\left(\mathrm{I}-\frac{l}{2 \pi}\right)^{n-\mathrm{I}}\right]
$$

St. Petersburg I 886 Juni 23 .
In folgender Tabelle sind die nach dieser Formel berechneten wahrscheinlichen Zahlen der Entfernungen von verschiedener Grösse mit denjenigen zusammengestellt, welche ich aus den Elementen der Planetenbahn direct erhalten habe.*)

\begin{tabular}{l|r|r|r}
\hline Entfernung & Zählg. & Rechn. & Diff. \\
\hline $0.0-0.4$ & 69 & 66.9 & $+\mathbf{2 . 1}$ \\
$0.5-0.9$ & 55 & 53.3 & $+\mathbf{1} .7$ \\
$1.0-1.9$ & 63 & 65.2 & -2.2 \\
$2.0-2.9$ & 36 & 32.4 & +3.6 \\
$3.0-3.9$ & 17 & 16.2 & +0.8 \\
$4.0-4.9$ & 6 & 8.2 & -2.2 \\
$5.0-5.9$ & 6 & 3.9 & +2.1 \\
6.0 u. mehr & 3 & 3.9 & -0.9 \\
& & 250.0 &
\end{tabular}

Es ist also in der That keine Spur von einer Condensation der Knoten vorhanden.

*) Da die Knoten-Längen in Zehntel Grad aufgeschrieben wurden, so bedeutet z. B. die erste Zahl, dass sich 69 solcher Punkte vorfinden, deren Entfernung von den nächstfolgenden $0.0,0.1,0.2,0.3$ oder 0.4 ist, also in den Grenzen 0.0 bis 0.45 eingeschlossen ist, für diese Grenzen gilt auch der theoretische Werth 66.9. Ebenso ist die Zahl 53.3 für das Intervall $0.45-0.95$ berechnet $\mathfrak{u}$. $s$. w.

Beobachtungen am Aequatoreal der Herzogl. Sternwarte zu Gotha.

\begin{tabular}{l|l|l|l|l|l|l|l|l|l|}
\hline I 886 & M.Z.Gotha & $\Delta \alpha$ & $\Delta \delta$ & Vgl. & $\alpha$ app. & $\log p . \Delta \mid$ &.$\delta$ app. & $\log p . \Delta \mid \operatorname{Red.ad}$ l.app. & $*$ \\
\hline
\end{tabular}

Comet $x 886 \mathrm{I}$.

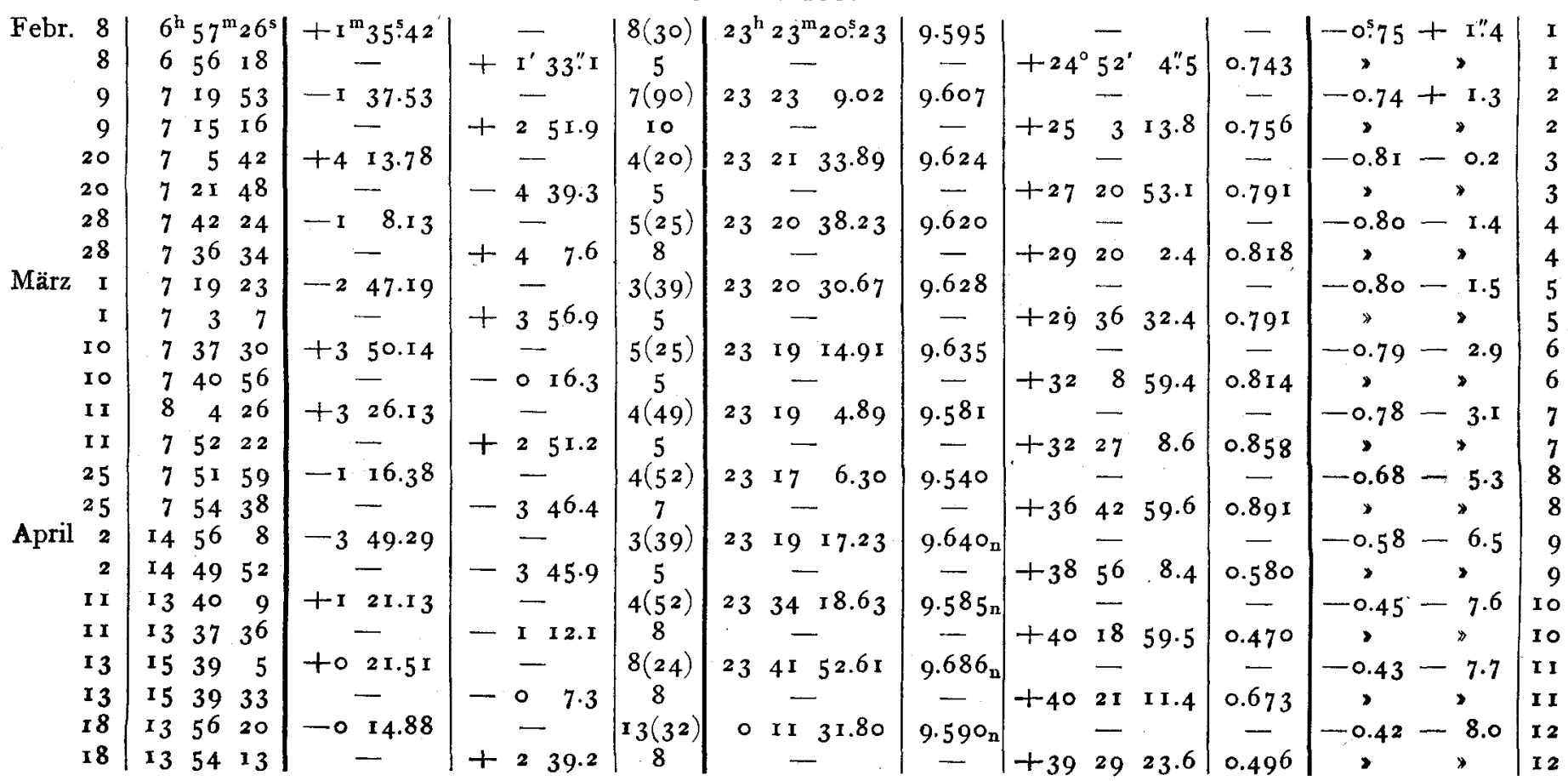

